\title{
LOGISTICS AND UPTAKE OF GENETIC TESTING FOR PATIENTS REFERRED FOR TELEGENETIC COUNSELLING FOR CANCER
}

\author{
Kate P Shane-Carson MS, LGC, Cortlandt Martin BS \\ The Ohio State University, Wexner Medical Center, Columbus, Ohio, USA
}

\begin{abstract}
Purpose: There is a dearth of information about the uptake of genetic testing after telegenetic (videoconference) counselling for hereditary cancer, which has been previously reported as a limitation of this service delivery model. Methods: We performed a review of the triage list for patients referred to The Ohio State University (OSU) from two community cancer centres for telegenetic counselling appointments from April 1, 2014 to May 31, 2016. Results: A total of 179 patients were referred for telegenetic counselling, and of these $62.6 \%(112 / 179)$ completed a 30-60 minute telegenetic counselling appointment. Of those counselled, $82.1 \%(92 / 112)$ completed genetic testing, $12.0 \%(11 / 92)$ of whom were found to have a pathogenic mutation. Of those with mutations, $45.5 \%(5 / 11)$ returned for a follow-up telegenetic counselling session to review results in more detail. In addition, 18.5\% (17/92) of patients tested had at least one variant of uncertain significance (VUS). Conclusions: The presence of a nurse/nurse practitioner with the patient at the remote site during the telegenetic counselling session may have facilitated higher completion rates for genetic testing compared to previous reports in the literature, and appropriateness of genetic testing in this method of providing genetic counselling is reflected in the rates of results identifying deleterious mutations and variants of uncertain significance.
\end{abstract}

Keywords: telegenetic counselling; genetic testing; telemedicine; hereditary cancer; genetic counselling

\section{Introduction}

Telegenetic counselling is a service delivery model in which genetic counselling is provided remotely by videoconference or a web-link that includes visual and audio access. ${ }^{1}$ Studies have shown that when telegenetic counselling services are compared to inperson genetic counselling, outcomes are similar in terms of patient satisfaction, anxiety, cancer worry, and knowledge. ${ }^{2-9}$ Cancer centres that do not currently employ a genetic counsellor are encountering an increasing need to provide genetic counselling services at their centres, due to requirements for accreditation and from insurance companies. The Commission on Cancer and the National Accreditation Program for Breast Centres both require access to cancer risk assessment and testing when indicated for program accreditation. ${ }^{10-11}$ Additionally, certain insurance providers, such as Cigna, Medical Mutual, and United Healthcare, now require genetic counselling in order to approve coverage for cancer genetic testing. ${ }^{12-14}$ As such, it is essential to increase access to genetic counselling, and the use of telegenetic counselling is considered a viable alternative to in-person counselling. ${ }^{2-4,9}$

In 2013 the Service Delivery Model Task Force (SDMTF) of the National Society of Genetic Counsellors (NSGC) surveyed members regarding their use of various service delivery models for genetic counselling. At that time, the results of the survey showed that $45.3 \%$ of respondents reported using multiple service delivery models (e.g. in-person, phone, telegenetic), but only $2.2 \%$ of respondents reported using telegenetic counselling often or always. ${ }^{1}$ Multiple studies have identified various advantages and barriers to utilising telegenetic counselling as a service delivery model. The common advantages have included: decreased travel distance for providers, patient preference of receiving genetic counselling at their local clinic, decreased wait time for an appointment, convenience, and increased access to care. ${ }^{3,4,15-17}$ The common disadvantages and barriers to care have included: a more labour-intensive process of scheduling appointments, difficulties with audio-visual quality, difficulties with sample collection and shipment, limited billing and reimbursement, requirement for equipment set-up, lack of support staff at the remote site, and inhibited rapport-building. ${ }^{1,2,4,15}$ 
Various methods of implementing telegenetic counselling for cancer genetic counselling have been employed and reported in the literature. In two studies the patients received telegenetic services at home. ${ }^{6,18}$ In other instances, telegenetic counselling services were provided with the genetic counsellor on-site with the patient, and the clinical geneticist meeting remotely via videoconference. ${ }^{8,9}$

The most common models, particularly in the United States, are those in which a genetic counsellor or clinical geneticist provides services via telegenetics to a patient at another healthcare facility, who may be either with or without support staff on-site with the patient. $2-4,7,17,19$ Most genetic counsellors in the United States have reported that they have been able to provide telegenetic counselling without a physician present during the session. ${ }^{1}$

In general, there is a dearth of information about the uptake of genetic testing after telegenetic counselling for hereditary cancer. Mette et al. (2016) surveyed underserved patients who had undergone either inperson genetic counselling or telegenetic counselling for cancer. Thirty-four percent of patients completed a survey, and $80 \%(n=95)$ of respondents reported having undergone genetic testing. Of the 119 survey respondents, $16 \%$ were found to have a pathogenic mutation and $10 \%$ were found to have a variant of uncertain significance (VUS) ${ }^{6}$ However, this study did not delineate if there was a difference in the uptake of genetic testing between the patients seen for in-person genetic counselling versus those seen for telegenetic counselling. Bradbury et al. (2016) evaluated patients who were offered telegenetic counselling for hereditary cancer at two remote sites. In that cohort, 74\% (61/82) of participants completed an initial telegenetic counselling session, and of those 67\% (41/61) proceeded with genetic testing. Of those tested $10 \%$ $(4 / 41)$ were found to have a pathogenic mutation. ${ }^{2}$ In a study evaluating uptake of $B R C A 1 / B R C A 2$ testing in patients randomised to either telephone genetic counselling or in-person genetic counselling, the telephone group was significantly less likely to proceed with genetic testing $(84.2 \%)$ than the in-person group $(90 \%){ }^{20}$ None of these studies discussed patientreported reasons for not proceeding with genetic testing.

In this paper, we describe the methods by which our telemedicine services are provided, with the presence of a nurse/nurse practitioner with the patient at the remote site how patients are triaged for clinic, the rate at which patients present to their telemedicine appointments, and the uptake and results of genetic testing in this patient population.

Description of our Telegenetic Model for Patient Scheduling and Provision of Services

\section{Collaborative Sites}

The Ohio State University (OSU) Division of Human Genetics has executed collaborative agreements with Holzer Cancer Centre (HCC) in Gallipolis, Ohio and Adena Cancer Centre (ACC) in Chillicothe, Ohio to provide telegenetic counselling services to their patients. HCC has a nurse and ACC has a nurse practitioner on-site who is dedicated to coordinating and attending the telegenetic counselling sessions at their respective cancer centres.

Referral Process

Patients at ACC and $\mathrm{HCC}$ are referred to genetic counselling at their centres through the telegenetic counselling program. At HCC, the patient has an initial appointment with the nurse associated with the program, and often also has a consultation with one of the physicians. At ACC, the patient has an initial appointment with the nurse practitioner affiliated with the program. During these initial consultations, the nurse/nurse practitioner obtains a complete medical history and collects 3-4 generations of family history information. They also attempt to obtain copies of any relevant pathology reports. These initial consultations are billed to the patient and/or their insurance. The nurse or nurse practitioner from ACC and HCC then sends the genetic counsellor at OSU the information from the initial consultation. The genetic counsellor reviews the information to determine if the patient meets standard guidelines for genetic testing. If the patient does not meet criteria, the genetic counsellor may request additional information about the patient and/or their family for further assessment of their eligibility for genetic testing. Patients who meet criteria for genetic testing are scheduled for a telegenetic counselling appointment. Patients who do not meet criteria for genetic testing are informed of this, but may schedule a telegenetic counselling appointment if they wish to discuss their familial risk factors in more detail. ACC and HCC schedule the telegenetic counselling appointments based on the availability of the genetic counsellor at OSU and the nurse/nurse practitioner at each site. Currently, both the genetic counsellor and nurse/nurse practitioner at each site routinely block one half-day per week for each site for telegenetic counselling appointments, in order to ensure 
consistent weekly clinic availability and expedite scheduling. However, there is open communication between sites to provide other appointment availability as needed in order to accommodate the patients.

Appointment Structure

The telegenetic appointments are typically structured as individual counselling sessions, although other relatives, spouses, and/or support persons may attend the appointment with the proband. ACC, HCC, and OSU each have a dedicated computer with a telephone for conference calling and a webcam for the telegenetic session. Currently, the sessions are performed via Cisco WebEx. Previously, sessions between ACC and OSU utilised the Cisco Jabber platform, but this software has been discontinued. The telegenetic appointments typically last 30-60 minutes. The genetic counsellor reviews the patient's medical and family histories, provides education about hereditary cancers, performs a risk assessment, and describes the genetic testing process. The nurse/nurse practitioner at each centre remains present with the patient during the duration of the telegenetic session to aid in remedying any technical issues that may occur. If the patient elects to proceed with genetic testing, the genetic counsellor obtains informed consent for the testing, and the nurse/nurse practitioner at each site aids in paperwork completion, sample collection, and sample shipping. The genetic counsellor then provides the patients with their genetic testing results by telephone. If the patient is found to have a pathogenic mutation or a VUS, they are offered the opportunity to return to their local cancer centre for a results appointment via telemedicine to review their genetic testing results in more detail.

\section{Other Logistics}

The genetic counsellor at OSU writes the initial consult letter and, if the patient proceeded with genetic testing, the results letter for the telegenetic counselling appointments. These letters are reviewed and signed by a collaborating physician in genetics at OSU. The completed letters are then sent to HCC and ACC for the respective nurse/nurse practitioner to sign, print on their clinic letterhead, and send to the patient and their physicians.

Billing

OSU bills ACC and HCC a flat rate for each telegenetic counselling session that includes the initial appointment and any follow-up appointments that may occur. The rate billed is $\$ 350$ USD, equivalent to 2.5 units of the 96040 CPT code for genetic counselling. ACC and
$\mathrm{HCC}$ are not billed by OSU for patients that are referred and triaged, but do not attend a telegenetic counselling session. The patients and/or their insurance are not billed for the telegenetic counselling appointments, other than for the initial consultations that are completed by the nurse/physician at HCC or the nurse practitioner at ACC.

\section{Methods}

This study was determined exempt from IRB review by the Office of Responsible Research Practices at The Ohio State University. In addition, the Ohio State University HIPAA Privacy Board granted this project a full waiver of HIPAA authorisation.

The genetic counsellor maintains a list of all patients referred for telegenetic counselling sessions at HCC and ACC. Information collected for tracking purposes includes: date of referral, whether the patient meets National Comprehensive Cancer Network (NCCN) genetic testing criteria, whether and what additional information was requested (i.e. pathology reports, additional family history information), whether the patient was recommended to schedule an appointment, whether the patient was scheduled for a telegenetic counselling appointment, whether they completed the appointment/did not show/rescheduled, whether genetic testing was ordered, when results were called to the patient, and when final letters were sent to the collaborating cancer centre. A review of this triage list was performed for patients referred from ACC and HCC for telegenetic counselling appointments from April 1, 2014 to May 31, 2016.

\section{Results}

A total of 179 patients, 118 from ACC and 61 patients from $\mathrm{HCC}$, were referred for telegenetic counselling appointments from April 1, 2014 to May 31, 2016. Of all patients referred, 62.6\% (112/179) completed a telegenetic counselling appointment.

Overall, 16.8\% (30/179) of referrals were marked "do not schedule" by the genetic counsellor because the patient did not meet NCCN criteria for genetic testing. Of all patients who met NCCN criteria for genetic testing, $72.5 \%(108 / 149)$ completed a telegenetic counselling session. In addition, 4 of the 30 patients who did not meet NCCN criteria for genetic testing elected to complete a telegenetic counselling session; however, none proceeded with genetic testing. Of all 
patients who met NCCN criteria for genetic testing, $3.4 \%(5 / 149)$ were scheduled but never showed for a telegenetic counselling appointment, 9.4\% (14/149) declined the telegenetic appointment, and 22/149 $(14.8 \%)$ did not return calls for scheduling a telegenetic appointment. A total of 112 of the 179 referred patients completed a telegenetic counselling session. Of these patients, 82.1\% (92/112) completed genetic testing. Finally, a total of $17.9 \%(20 / 112)$ of patients seen for an initial telegenetic counselling session did not complete genetic testing. The reasons for this are listed in Table 1.

Table 1. Patients seen for an appointment who did not complete testing $(\mathrm{n}=20)$.

\begin{tabular}{|c|l|}
\hline $\mathbf{n}=$ & Reason not tested \\
\hline 3 & $\begin{array}{l}\text { Testing was cancelled because it was not } \\
\text { covered by insurance }\end{array}$ \\
\hline 4 & $\begin{array}{l}\text { Patients triaged "do not schedule" because } \\
\text { they did not meet NCCN criteria, patient } \\
\text { elected to be seen for appointment, testing } \\
\text { not ordered }\end{array}$ \\
\hline 7 & $\begin{array}{l}\text { A relative was a better person in whom to } \\
\text { initiate testing }\end{array}$ \\
\hline 3 & $\begin{array}{l}\text { Patient declined testing due to low risk of } \\
\text { identifying a mutation }\end{array}$ \\
\hline 1 & $\begin{array}{l}\text { Patient met NCCN criteria, sample sent, } \\
\text { patient then declined and cancelled testing }\end{array}$ \\
\hline 1 & $\begin{array}{l}\text { Patient had a previous negative result, and } \\
\text { additional genetic testing was not } \\
\text { recommended }\end{array}$ \\
\hline 1 & $\begin{array}{l}\text { Recommended obtaining results from } \\
\text { relative with a positive result in order to } \\
\text { offer targeted testing to the patient }\end{array}$ \\
\hline
\end{tabular}

Of the patients who completed genetic testing, $12.0 \%(11 / 92)$ were found to have a pathogenic mutation. The positive results are illustrated in Table 2. Of note, one patient was found to have two pathogenic mutations (single mutations in BRCAI and CHEK2), and one patient was found to have a monoallelic mutation in MUTYH. Of those who were found to have mutations, $45.5 \%(5 / 11)$ returned for a follow-up telegenetic counselling session to review their results in more detail. In addition, 18.5\% (17/92) of all patients tested had at least one variant of uncertain significance (VUS). Of these 17 patients, 2 also had a pathogenic mutation. One patient had a $P A L B 2$ pathogenic mutation with a BRIPI VUS, and the other patient had a $R A D 51 C$ pathogenic mutation with an
$A T M$ VUS. Also, one patient had a total of 2 variants of uncertain significance (in BRCAI and BRIPI).

Table 2. Pathogenic mutations identified in patients who completed testing $(\mathrm{n}=11)$.

\begin{tabular}{|l|c|}
\hline Pathogenic Mutation & $\mathbf{n}=$ \\
\hline$A T M$ & 1 \\
\hline BRCA1 & 1 \\
\hline BRCA1 and CHEK2 & 1 \\
\hline BRCA2 & 1 \\
\hline BRIP1 & 1 \\
\hline CHEK2 & 2 \\
\hline MUTYH (monoallelic mutation) & 1 \\
\hline PALB2 & 2 \\
\hline RAD51C & 1 \\
\hline
\end{tabular}

\section{Discussion}

Within the cohort of patients referred for telegenetic counselling for cancer, uptake for the telegenetic counselling appointment and genetic testing was high. Most patients $(82.6 \%)$ who proceeded with genetic testing had at least a 7-gene high risk cancer panel. Of the 92 patients who completed genetic testing, 12\% were found to carry mutations, and $18.5 \%$ were found to have variants of uncertain significance.

In this cohort, $72.5 \%$ of patients referred, who met NCCN guidelines for cancer genetic testing, had an initial telegenetic counselling session. This show-rate is comparable to that of Bradbury et al., who reported $74 \%$ of patients who met NCCN guidelines presented for an initial telegenetic counselling session. ${ }^{2}$ There are various reasons why patients may not have presented to an appointment. Some of these patients had multiple appointments scheduled, but had recurrent appointment cancellations due to illness or transportation problems. Some patients may also have decided after their baseline consultation with the nurse/nurse practitioner that they were no longer interested in genetic counselling and testing. The logistics of coordinating an appointment with the patient, genetic counsellor, and nurse/nurse practitioner at the remote site may also have been a barrier to scheduling the telegenetic consultation.

In the study cohort, it was possible to evaluate the uptake of genetic testing amongst patients who completed an initial telegenetic counselling session. Of all patients seen, $82.1 \%$ completed genetic testing. A $67 \%$ testing rate was reported by Bradbury et al. in their 
similar cohort. ${ }^{2}$ The rate observed here is comparable with the genetic testing rate of $80 \%$ reported by Mette et al.; however, the rate of testing reported by that group was for all patients seen, and did not distinguish testing rates between those seen for a telegenetic counselling session versus those seen for an in-person appointment. ${ }^{6}$ Since data are limited regarding the uptake of genetic testing in telegenetic counselling appointments, we also compared our results to those of a study of patients who completed telephone genetic counselling for hereditary cancer, which showed that $84.2 \%$ of patients completed genetic testing. ${ }^{20}$ Our completion rate for genetic testing may have been higher because of the presence of a nurse/nurse practitioner with the patient to help facilitate the blood draw at the time of the telegenetic appointment. Reasons for not completing genetic testing after the telegenetic counselling session are listed in Table 1.

Also evaluated were the types of results obtained from patients who received genetic testing in the cohort, to see if results were comparable to other comprehensive cancer clinics. A recent paper evaluating mutation prevalence amongst the first 10,000 patients receiving next-generation panel testing, showed that $9.0 \%$ of their cohort had a pathogenic or likely pathogenic mutation identified. ${ }^{21}$ Bradbury et al. showed that patients who had telegenetic counselling and genetic testing had a mutation positive rate of $10 \%$, which is quite similar to this study $(12 \%){ }^{2}$ Mette et al. reported that $16 \%$ of patients tested positive; however, their results did not indicate whether these numbers were obtained by chart review or patient report. ${ }^{6}$ This study also evaluated the VUS rate within the cohort. The VUS rate reported by Susswein et al. ranged from $22.7 \%$ for Caucasian individuals to $39.7 \%$ in African Americans, with smaller panels showing lower VUS rates than larger gene panels. ${ }^{21}$ Of all of the patients who completed genetic testing within the current study cohort, $82.6 \%$ had at least a 7 -gene high risk cancer panel, and $18.5 \%$ of patients had at least one VUS. This number was lower than the expected VUS-rate from panel testing of 22.7-39.7\% ${ }^{21}$ However, the proportion of patients tested with a smaller gene panel may have been lower than those reported by Susswein et al., resulting in the lower VUS-rate. Mette et al. reported a lower VUS rate of $16 \%$; however, this population only completed BRCA1 and BRCA2 gene testing. ${ }^{6}$ Therefore, Mette et al.'s VUS rate would be expected to be lower, as the patients were not tested with a gene panel.
No literature reporting follow-up telegenetic counselling for patients who are found to have a pathogenic mutation could be found. In the study cohort, 11 patients tested positive for a pathogenic mutation. All patients who were found to have a pathogenic mutation were offered the opportunity for a follow-up telegenetic counselling session to review their results in more detail, and approximately half of these patients $(5 / 11)$ presented for the session. Reasons why the other patients did not return for a follow-up consultation are unknown. Some possible reasons could include: they may not have had further questions, they may not have chosen to bring family members with them, or they may have felt that their local healthcare practitioners could address their questions and concerns.

Our model of seeing patients is consistent with other models typically reported in the US, with the genetic counsellor working off-site to provide the telegenetic counselling services to a patient who is at a remote medical facility. ${ }^{2-4,7,17,19}$ As previous literature has suggested, the process of scheduling appointments is more labour-intensive, since schedules must be coordinated between the availability of the patient, as well as the availability of the genetic counsellor and the support staff at both medical facilities. ${ }^{7}$ However, under OSUs current telegenetic counselling model, appointments could be offered consistently on a weekly basis, with greater feasibility to offer other appointment times as needed. This model allowed flexibility to accommodate patients for appointments via telegenetic counselling in a more timely fashion than when the genetic counsellor travelled to those remote sites once per month for in-person counselling sessions. It is believed that the model of having a dedicated nurse or nurse practitioner at each of the remote sites during the telegenetic counselling sessions helped to address some of the previously-stated limitations of telegenetic counselling, including lack of support staff at the remote site and difficulties with sample collection and shipment. ${ }^{1,6,7,15}$ Also, since the nurse/nurse practitioner met with the patient for an initial face-to-face consultation at the patient's local cancer centre, and since the same nurse/nurse practitioner was present with the patient during the telegenetic counselling session, their existing rapport may have served to increase patient attendance for the telegenetic counselling session. Furthermore, having the nurse/ nurse practitioner present during the session provideda means of fixing any technical difficulties and ensuring 
sample collection and shipment occur at the time of the telegenetic counselling session. Since the genetic testing in this setting does not require an extra appointment on the part of the patient, the assistance of a local provider may lead to increased uptake of genetic testing.

This study is the first to examine the reasons why patients who had telegenetic counselling for hereditary cancer may not have proceeded with genetic testing. Of note, none of the reasons included barriers to sample collection. In most cases, those who were not tested either did not meet criteria (the 4 patients that elected to come only to discuss their familial risk of cancer) or a relative was determined to be a better person to initiate testing in the family ( $7 / 20$ of patients not tested).

\section{Conclusion}

The presence of a dedicated nurse/nurse practitioner at each of the remote sites facilitates the triage process through the collection of a detailed medical and family history. Furthermore, having the nurse/nurse practitioner present with the patient at the remote site during the telegenetic counselling session facilitates sample collection and shipping. Although previous reports in the literature note that one of the barriers to telegenetic counselling is the facilitation of sample collection for genetic testing, the processes for sample collection have not been previously described. Thus, the presence of a dedicated practitioner with the patient may be one effective means of addressing this limitation. The appropriateness of genetic testing in this cohort is reflected in the rate of positive results and the VUS-rate, which is consistent with rates that would be expected in genetic testing for hereditary cancer. Although the model of providing telegenetic counselling and testing addresses some of the barriers to providing genetic counselling via telemedicine, outcomes of this service delivery model have not been evaluated in other practice settings (i.e. cardiogenetics, prenatal, paediatrics, or other specialties). In addition, data about the initial referrals at the collaborating cancer centres are not available, and therefore the percentage of initial referrals at the cancer centres who complete the pre-genetic counselling appointment is unknown. Further, there are no data from the cancer centres about the wait times from initial referral to initial appointment at the cancer centres to the telegenetic counselling appointment. It would be valuable to compare this process to other triage methods, in order to define the most appropriate model that could be implemented for telegenetic services that would increase access to care in a timely manner.

\section{Corresponding author: \\ Kate P Shane-Carson \\ Clinical Cancer Genetics \\ 2012 Kenny Road \\ Columbus, OH 43221 \\ Phone: 614-293-2298 \\ Fax: 614-293-2314 \\ E-mail: kate.shane@osumc.edu}

Conflict of Interest. The authors declare no conflicts of interest.

\section{References}

1. Cohen SA, Marvin ML, Riley BD, et al. Identification of genetic counselling service delivery models in practice: a report from the NSGC Service Delivery Model Task Force. $J$ Genet Counsell 2013;2(4):411-421. DOI:10.1007/s 10897-013-9588-0.

2. Bradbury A, Patrick-Miller L, Harris D, et al. Utilizing remote real-time videoconferencing to expand access to cancer genetic services in community practices: a multicenter feasibility study. J Med Internet Res 2016;18(2):e23. DOI:10.2196/jmir.4564.

3. Buchanan AH, Datta S, Skinner CS, et al. Randomized trial of telegenetics vs. in-person cancer genetic counselling: cost, patient satisfaction and attendance. $J$ Genet Counsell 2015;24(6):961-970.

4. Hilgart JS, Hayward JA, Coles B, Ireldale R. Telegenetics: a systematic review of telemedicine in genetics services. Genet Med 2012;14(9):765776.

5. McDonald E, Lamb A, Grillo B, Lucas L, Miesfeldt S. Acceptability of telemedicine and other cancer genetic counselling models of service delivery in geographically remote settings. J Genet Counsell 2014;23(2):221-228.

6. Mette LA, Saldívar AM, Poullard NE, et al. Reaching high-risk underserved individuals for cancer genetic counselling by video- 
teleconferencing. J Community Support Oncol 2016;14(4):162-168.

7. Portigal-Tood C. Impact of telegenetics and alternative models of care on hereditary cancer genetic counselling in British Columbia. Estudos 2014; Goiania,41(especial):13-33.

8. Zilliacus E, Meiser B, Lobb E, et al. Women's experience of telehealth cancer genetic counselling. J Genet Counsell 2010;19(5):463472. DOI: $10.1007 / \mathrm{s} 10897-010-9301-5$.

9. Zillacus EM, Meiser B, Lobb EA, et al. Are videoconferenced consultations as effective as face-to-face consultations for hereditary breast and ovarian cancer genetic counselling? Genet Med 2011;13(11):933-941.

10. American College of Surgeons Commission on Cancer. Cancer program standards: ensuring patient-centered care. (2016) Available at: https://www.facs.org/ /media/files/quality\%20pr ograms/cancer/coc/2016\%20coc $\% 20$ standards $\% 2$ 0manual_interactive\%20pdf.ashx accessed 5May 2018.

11. American College of Surgeons Commission on Cancer. NAPBC standards manual. (2014) Available at:

https://www.facs.org/ /media/files/quality $\% 20 \mathrm{pr}$ ograms/napbc/2014\%20napbc $\% 20$ standards $\% 20$ manual.ashx accessed 5May 2018.

12. Cigna. Genetic testing and counselling program: genetic counselling requirement. (2018)

Available at: https://www.cigna.com/healthcareprofessionals/resources-for-health-careprofessionals/genetic-testing-and-counselingprogram accessed 9 May 2018.

13. Medical Mutual. Prior approval and investigational services. Available at: https://provider.medmutual.com/pdf/priorapprova 1list.pdf accessed 9 May 2018.

14. United HealthCare Services. Genetic testing for hereditary breast and/or ovarian cancer syndrome (HBOC). Medical Policy 04/01/2017. (2017) Available at: https://www.oxhp.com/secure/policy/genetic test ing for_hereditary breast_and_or_ovarian_cance r_syndrome hboc.pdf accessed 9 May 2018.

15. Cohen SA, Huziak RC, Gustafson S, Grubs RE. Analysis of advantages, limitations, and barriers of genetic counselling service delivery models. $J$ Genet Counsell 2016;25(5):1010-1018. DOI:10.1007/s10897-016-9932-2.
16. Zilliacus EM, Meiser B, Lobb E, et al. The virtual consultation: practitioners' experiences of genetic counselling by videoconferencing in Australia. Telemed J e-Health 2010;16(3):350357. DOI:10.1089/tmj.2009.0108.

17. Scheuner MT, Marshall N, Lanto A, et al. Delivery of clinical genetic consultative services in the Veterans Health Administration. Genet Med 2014;16(8):609-619.

18. Meropol NJ, Daly MB, Vig HS, et al. Delivery of internet-based cancer genetic counselling services to patients' homes: a feasibility study. $J$ Telemed Telecare 2011;17(1):36-40.

DOI: $10.1258 /$ jtt.2010.100116.

19. Elliott AM, Mhanni AA, Marles SL, et al. Trends in telehealth versus on-site clinical genetics appointments in Manitoba: a comparative study. J Genet Counsell 2012;21(2):337-344.

20. Butrick M, Kelly S, Peshkin BN, et al. Disparities in uptake of BRCA1/2 genetic testing in a randomized trial of telephone counselling. Genet Med 2015;17(6):467-475.

21. Susswein LR, Marshall ML, Nusbaum R, et al. Pathogenic and likely pathogenic variant prevalence among the first 10,000 patients referred for next-generation cancer panel testing. Genet Med 2015;18(8):823-832.

DOI:10.1038/gim.2015.166. 